\title{
Génesis, concepto y desarrollo del capital intelectual en la economía del conocimiento: Una reflexión sobre el Modelo Intellectus y sus aplicaciones
}

\author{
EDUARDO BUENO, $\mathrm{M}^{\mathrm{a}}$ PAZ SALMADOR y CARLOS MERINO \\ Departamento de Organización de Empresas \\ e Instituto Universitario de Investigación IADE \\ UNIVERSIDAD AUTÓNOMA DE MADRID \\ e-mail: eduardo.bueno@uam.es; maripaz.salmador@uam.es; carlos.merino@uam.es
}

\begin{abstract}
RESUMEN
En el entorno socio-económico actual de la Economía del Conocimiento, la principal fuente de ventaja competitiva sostenible de las organizaciones reside fundamentalmente en sus activos de naturaleza intangible, reflejados en su Capital Intelectual. En la última década del siglo XX, comenzó a desarrollarse la principal literatura de Dirección del Conocimiento y Capital Intelectual en las organizaciones, y con ella surgieron los primeros esfuerzos de evaluación de este último concepto. El presente trabajo muestra, en primer lugar, el origen, justificación y desarrollo del Capital Intelectual, para pasar seguidamente a destacar los principales modelos. A continuación, se analiza el concepto de Capital Intelectual, y seguidamente las principales aplicaciones y experiencias desarrolladas de uno de los modelos presentados, el Modelo Intellectus. Finalmente, se recogen las conclusiones del trabajo y posibles líneas de investigación a desarrollar sobre el tema.
\end{abstract}

Palabras clave: Activos intangibles; capital intangible; capital intelectual; dirección del conocimiento; modelos del capital intelectual; modelo Intellectus.

\section{Origins, Concept and Developments of Intellectual Capital in the Knowledge Economy: Intellectus Model and its Main Applications}

\begin{abstract}
The main source of competitive sustainable advantage, in the current socio-economic environment of the Knowledge Economy, resides basically in the Intellectual Capital. The main literature on Knowledge Management and Intellectual Capital was developed in the last decade of the 20th century, giving rise to the initial attempts for evaluating the latter concept. This paper examines firstly the origin and development of the Intellectual Capital and discusses the chief existing models. Then, the concept of Intellectual Capital is analysed together with the main applications and implementations of a particularly relevant model, the Intellects model. Finally, some conclusions and future research extensions are presented.
\end{abstract}

Keywords: Intangible Capital; Intellectual Capital; Intellectual Capital Models; Intellectus Model.

Clasificación JEL: M1.

Artículo recibido en mayo de 2008 y aceptado para su publicación en julio de 2008.

Artículo disponible en versión electrónica en la página www.revista-eea.net, ref. ə-26203. 


\section{INTRODUCCIÓN}

Hace casi cuatro décadas, Bell (1973) constataba, por una parte, la evolución de la sociedad basada en la agricultura hacia la sociedad industrial, y por otra, la transformación de ésta última en la sociedad post-industrial. Mientras que en la economía agrícola el recurso principal era la tierra y en la economía industrial fue el capital físico, actualmente, el conocimiento se ha convertido en el recurso estratégico por excelencia. Este planteamiento deriva de la aportación precursora de Marshall (1890) sobre aquél como cuarto factor o agente de producción así como de las contribuciones que le siguieron gracias a las ideas de Drucker (1965) y Machlup (1980), entre otros.

En consecuencia, la llegada de la sociedad de la información y su evolución hacia la del conocimiento ha situado a los recursos intangibles basados en conocimiento en una de las fuentes principales de creación de ventaja competitiva sostenible de la empresa y de generación de valor y desempeño futuro de las organizaciones (Amir y Lev, 1996; Aboody y Lev, 1998; Edvinsson, 1997; Hendrick, 1961; Lev, 1989, 2001). En este contexto surge el concepto del Capital Intelectual, como perspectiva estratégica de la "cuenta y razón" de los intangibles de la organización (Bueno, 2002).

Desde la emergencia del citado concepto, son varios los esfuerzos vinculados que se han venido llevando a cabo por la comunidad académica y empresarial con objeto de evaluar, desde una perspectiva estática, y dirigir, desde una perspectiva dinámica, el conocimiento relevante desde el punto de vista estratégico para una organización tanto en su dimensión de presente como de futuro (Andriessen, 2004; Moon y Kym, 2006; Kristandl y Bontis, 2007). El presente trabajo realiza una revisión del estado de la situación con objeto de sintetizar algunos de los principales desarrollos acometidos y poner en relieve futuros retos y líneas de investigación. Se pone especial énfasis en el Modelo Intellectus y en su contraste y aplicación en diversas organizaciones en España.

El desarrollo del artículo se estructura en torno a las siguientes partes diferenciadas que señalamos a continuación. En primer lugar, se muestra el origen, justificación y desarrollo del Capital Intelectual, para pasar seguidamente a presentar los principales modelos. A continuación, se analiza y propone un el concepto de Capital Intelectual después se presenta uno de los modelos de mayor difusión, en el ámbito iberoamericano: el Modelo Intellectus, considerando sus aplicaciones y experiencias desarrolladas. Finalmente, se recogen las conclusiones del trabajo y las posibles líneas de investigación futura abiertas.

\section{JUSTIFICACIÓN, ORIGEN Y DESARROLLO DEL CAPITAL INTELECTUAL}

Según Edvinsson y Malone (1997), el Capital Intelectual hace referencia al valor resultante del conjunto de intangibles creados por la empresa. Se define así como un Capital Intangible por su naturaleza, basado en el conocimiento o en el intelecto humano y desarrollado en la organización. En consecuencia ha sido percibido co- 
mo un capital oculto o invisible, dado que la contabilidad, con sus principios y prácticas de base jurídica-financiera, no lo venía considerando ni reflejando en las cuentas anuales. Incluye, por lo tanto, un conjunto de activos como son los sistemas organizativos, marcas, bases de datos, patentes, relaciones con los clientes, entre otros. Estos activos, como señala Lev (2001), responden a procesos de creación de valor, de innovación o de base cognitiva de la $\mathrm{I}+\mathrm{D}$, de las prácticas y políticas organizativas o de dirección y de la función de las personas como poseedoras de conocimiento, a la vez que es consecuencia de determinada estrategia que pone en acción el conocimiento poseído en la organización (Davenport y Prusak, 1998).

La justificación de la aparición del Capital Intelectual se puede argumentar de acuerdo con dos líneas explicativas. La primera y la más mediata, a la vez que mediática, hace referencia a la publicación de los primeros informes o estudios de Capital Intelectual y su difusión en medios de comunicación. La segunda se relaciona con la preocupación de los analistas financieros y economistas sobre cómo explicar las diferencias valorativas de las empresas cotizadas en Bolsa, intensivas en inversiones de I+D, entre su valor de mercado y su "valor en libros".

La primera explicación, siguiendo a Roos et al. (1998), nos lleva a recordar, entre otras aportaciones, lo publicado en el periodo 1993-1998: el informe anual de Skandia de 1992, elaborado por L. Edvinsson como responsable de Capital Intelectual de la compañía; los comentarios realizados en Forbes (25-10-1993), Los Angeles Times (1995), Fortune (3-10-1994) e Industry Week (19-2-1996) por R. Karlgeard (director de Forbes Asap); la entrevista a M.H. Wallman (presidente de la SEC de New York), realizada por M. S. Malone (columnista de los Angeles Times); la conferencia pronunciada por K. Bradley en la Real Sociedad de Artes de Londres el 21 de octubre de 1996; o la obra de Stewart (1997), el "Capital Intelectual como nueva riqueza de las naciones".

La segunda explicación del Capital Intelectual aparece vinculada como fundamento teórico para la búsqueda de una exégesis del ratio elevado entre el valor de mercado y el valor contable de las empresas con mayor volumen de cotización y más apreciadas de la Bolsa de New York durante las décadas ochenta y noventa del pasado siglo (Abramovitz y David, 1996; Amir y Lev, 1996; Aboody y Lev, 1998). Ratio del Capital Intelectual que se asemeja a la $Q$ de Tobin (1969), tal y como señalan Cetin (2000) y Megna y Klock (1993). Esta línea de investigación muestra su interés por definir el capital intangible existente en las empresas que más valoriza el mercado o, en otras palabras, por la existencia de unos activos de naturaleza intangible, consecuencia de la puesta en acción de actividades basadas en conocimiento y generadoras de dicho valor (Saint Onge, 1996; Sveiby, 1997; Bueno, 1998; Lev y Zarowin, 1999). Este enfoque basado en los intangibles creadores de valor fue iniciado por Ball y Brown (1968). En suma, esta justificación pone de manifiesto la existencia de unos activos intangibles, no informados adecuadamente, o de un Capital Intangible que en combinación con el capital físico-financiero tradicional es fuente de creación de valor en la economía de las organizaciones actuales (Lev y Sougiannis, 1996). 
La génesis del nuevo concepto de Capital Intelectual, expresión que nada más conocerse se difundió rápidamente en las comunidades académicas y profesionales relacionadas con el asunto, aparece asociada a la evolución de la economía del conocimiento. Basándonos en Kendrick (1961), se puede afirmar que el concepto de Capital Intelectual fue introducido por primera vez en el siglo XIX por el economista alemán F. List (1841), quien lo define como aquél referido a las naciones, o a la humanidad, por la acumulación de los descubrimientos, invenciones, esfuerzos, etc..., de las generaciones precedentes. De otra parte, se van avanzando conceptos, aspectos y enfoques asociados al nuevo término en desarrollo por otros economistas ingleses, caso de Senior (1836), quien introduce una primera visión del "capital relacional"; de Sidgwick (1883), que siguiendo la "tradición fabiana" incorpora un primer concepto de "capital social"; y de J. Bentham (1789) y J.S. Mill (1848), quienes proponen fundamentos de base psicológica y ética así como de libertad, para justificar el desarrollo del valor intangible del "utilitarismo". A esta génesis contribuye la aportación del sociólogo francés A. Comte (1848) quien, con su dinámica social, pretende salvar la unidad orgánica del conocimiento humano. Este autor tuvo gran influencia, junto a Sidgwick, en el pensamiento de Alfred Marshall, cuando en sus Principios de Economía (1890) declara que el conocimiento es el factor de producción que valoriza, por encima de los otros factores clásicos, la creación de bienes y servicios o la transformación económica. Con él se inicia la actual economía del conocimiento (Bueno, 2002).

En consecuencia, el Capital Intelectual ha ido evolucionando gracias a diversas aportaciones, entre las que se incluyen: el "utilitarismo ético" de Bentham; la filosofía económica del nacionalismo económico alemán de List; la filosofía moral y ética de Mill y Sidgwick, creadora del "capital intangible social"; la teoría del valor de cambio de Senior, base del "capital relacional"; el "evolucionismo y positivismo" de Comte, que fundamenta que el conocimiento se convierte, como dijera Marshall, en la máquina de producción más potente y que nos permite someter a la naturaleza, para añadir que la organización ayuda al conocimiento y tiene muchas formas; terminando, en fechas más cercanas, en una visión financiera o de "cuenta y razón" que pretende explicar el por qué del ratio elevado observado en las últimas décadas del siglo pasado entre el valor de mercado y el valor contable de las organizaciones o de las empresas que cotizan en Bolsa.

La existencia del Capital Intelectual comentada requiere el desarrollo de herramientas nuevas y adecuadas para medir, informar y dirigir estratégicamente y de una manera sistemática e integrada los recursos organizativos basados en el conocimiento que lo conforman (Mouritsen, Larsen y Bukh, 2001; Petty y Guthrie, 2000). Son varias las investigaciones que han realizado importantes esfuerzos en esta línea, centradas en la identificación, estructuración y evaluación de los citados recursos. Abordamos este aspecto en el siguiente epígrafe. 


\section{PRINCIPALES MODELOS DEL CAPITAL INTELECTUAL}

A mediados de la década de los noventa comienzan a surgir una serie de modelos de medición y gestión del Capital Intelectual en las organizaciones. En la Figura 1 se recoge la evolución del Capital Intelectual a través de la consideración de principales modelos que han tratado la cuestión.

En dicha figura se pueden identificar en el tiempo transcurrido desde la década de los noventa del siglo pasado hasta el presente siglo XXI tres enfoques principales. El primero que podría ser denominado "financiero-administrativo" (1992-2001), de clara influencia contable, como se verá más adelante; el segundo o "enfoque estratégico corporativo" (1997-2001); y el tercero o "enfoque social-evolutivo" (2000-2005), que inicia el desarrollo del Capital Intelectual para los años próximos.

Como se puede observar en esta trayectoria temporal, se han caracterizado los tres enfoques por la intersección de las dos dimensiones siguientes: a) en ordenadas, los modelos se han clasificado de menor a mayor número de componentes principales o "capitales básicos" considerados en su estructura explicativa: b) en abscisas, se han ordenado de menor a mayor complejidad o diversidad informativa, en coherencia con la propia evolución del entorno de simple a complejo, al que pretende dar "cuenta y razón" el Informe de Capital Intelectual (Lev, 2001 y Meritum Project, 2002).

En consecuencia, en el Cuadro 1 se han recogido los modelos principales integrantes de los tres enfoques propuestos. En el primer enfoque se presentan ocho modelos pioneros, los cuales responden a distintas demandas para medir e informar sobre el capital intangible o sobre los activos intangibles de las organizaciones que el mercado reconoce, pero no la contabilidad tradicional; preocupación que ha sido justificada en el epígrafe precedente. El segundo en el que se muestran siete modelos, inicia el proceso de madurez del concepto, al aparecer una perspectiva estratégico-corporativa en los modelos y en los que, como se observa en el Cuadro 2, se va produciendo una armonización de los componentes o "capitales" que lo integran, superando la heterogeneidad y ausencia de lógica teórica de la etapa inicial. Dentro de este enfoque estratégico, es interesante mencionar asimismo las investigaciones de autores como Reed, Lubatkin, y Srinivasan (2006) y Kristandl y Bontis (2007), quienes analizan el concepto del Capital Intelectual desde la perspectiva de la Teoría de los Recursos y Capacidades (Wernerfelt, 1984; Barney, 1991). Por último, el tercer enfoque ofrece con los cuatro modelos propuestos, de una parte, una madurez del concepto y, de otra, la resultante de modelos más evolucionados y con preocupación por componentes o capitales más "dinámicos" como son el "social", "cultural", de "innovación" o de "emprendimiento", entre otros. En estos últimos modelos hay que destacar el Modelo Intellectus (Bueno, E.-CIC, 2003), el KMCI (McElroy, 2001) y el NOVA (Camisón et al., 2000). Algunos de los citados modelos son analizados por Andriessen (2004), centrándose fundamentalmente en los motivos y soluciones que plantean los mismos. 


\section{FIGURA 1}

Evolución del capital intelectual: enfoques principales.

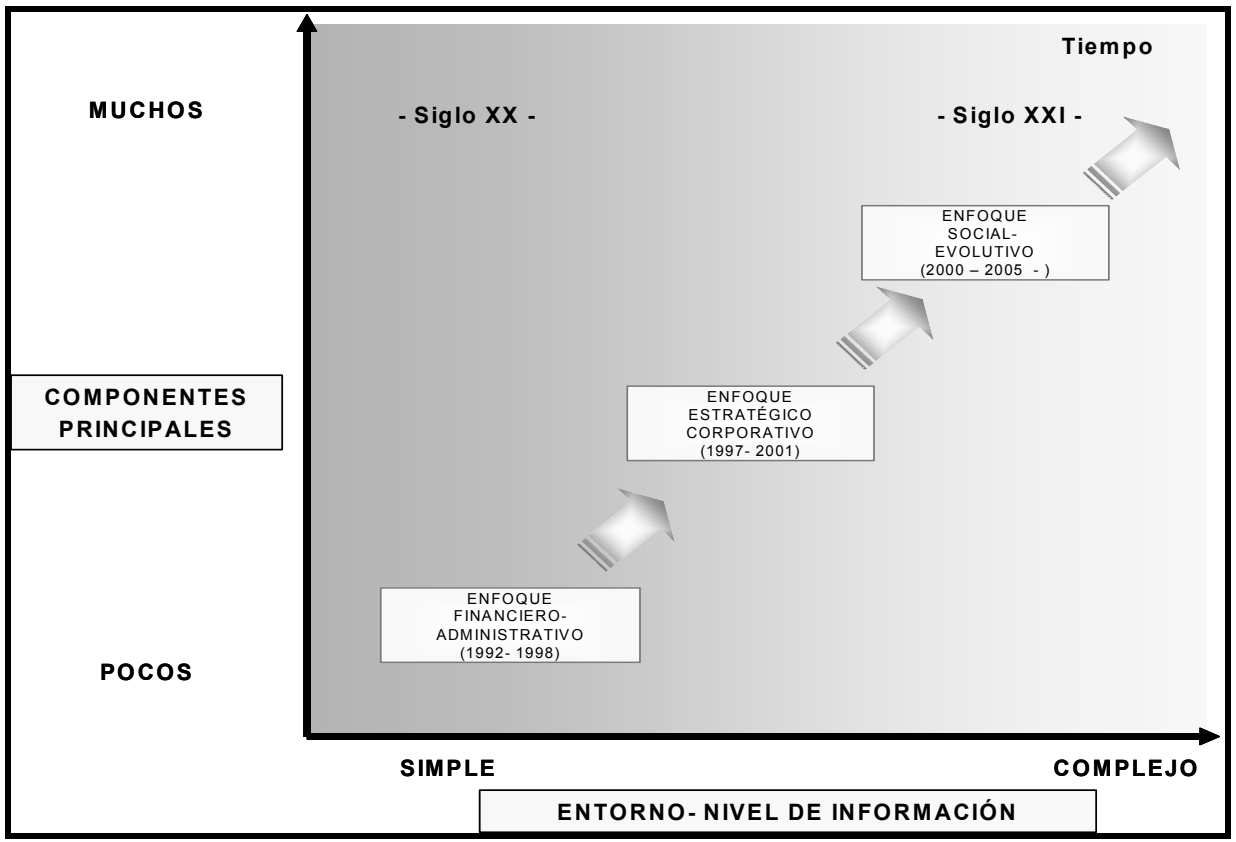

Fuente: Bueno (2005).

CUADRO 1

Modelos principales de capital intelectual según enfoques de análisis.

\begin{tabular}{|c|c|c|}
\hline $\begin{array}{c}\text { Enfoque } \\
\text { Financiero - Administrativo } \\
(1992-1998)\end{array}$ & $\begin{array}{c}\text { Enfoque } \\
\text { Estratégico - Corporativo } \\
(1997-2001) \\
\end{array}$ & $\begin{array}{c}\text { Enfoque } \\
\text { Social - Evolutivo } \\
(2000-2005)\end{array}$ \\
\hline $\begin{array}{l}\text { - Navigator Of Skandia (Skandia, } \\
1992 \text { y Edvinsson, 1997): Suecia. } \\
\text { - Technology Broker (Brooking, 1996): } \\
\text { Reino Unido. } \\
\text { - Canadian Imperial Bank of Com- } \\
\text { merce (Saint Onge, 1996): Ca- } \\
\text { nadá. } \\
\text { - University Of Western Ontario } \\
\text { (Bontis, 1996): Canadá. } \\
\text { - Intangible Assets Monitor (Svei- } \\
\text { by 1997b): Australia. } \\
\text { - Stewart, T.A. (1997): EE.UU. } \\
\text { - Dow Chemical (Dow Chemical, } \\
\text { 1998): EE.UU. }\end{array}$ & $\begin{array}{l}\text { - Atkinson, Waterhouse y Wells } \\
\text { (1997): USA. } \\
\text { - Roos, Roos, Edvinsson y Dra- } \\
\text { gonetti (1998): Suecia-Reino } \\
\text { Unido. } \\
\text { - Intellectual Capital Model (Bon- } \\
\text { tis, 1998). } \\
\text { - Intelect, I.U. Euroforum Esco- } \\
\text { rial (Bueno, Jericó y Salma- } \\
\text { dor, 2000): España. } \\
\text { - Dirección Estratégica Por Com- } \\
\text { petencias: Capital Intangible } \\
\text { (Bueno, 2002): España. } \\
\text { - Cluster Del Conocimiento (2000): } \\
\text { España. } \\
\text { - IICBS (Viedma, 2001): España. }\end{array}$ & $\begin{array}{l}\text { - American Society For Training } \\
\text { And Development (Astd, 2000): } \\
\text { USA. } \\
\text { - NOVA (Camisón, Palacios y De- } \\
\text { vece, 2000): España. } \\
\text { - KMCl (Mc Elroy, 2001): USA. } \\
\text { - Intellectus (Bueno - CIC, 2003): } \\
\text { España. } \\
\text { "Otros en elaboración". }\end{array}$ \\
\hline $\begin{array}{l}\text { Componentes o "capitales" no } \\
\text { armonizados: activos } \\
\text { intangibles y competencias }\end{array}$ & $\begin{array}{l}\text { Componentes o "capitales } \\
\text { armonizados: humano } \\
\text { estructural y relacional }\end{array}$ & $\begin{array}{l}\text { Componentes o "capitales" } \\
\text { armonizados- evolucionados }\end{array}$ \\
\hline
\end{tabular}

Fuente: Bueno, E.-CIC (2003) y elaboración propia. 


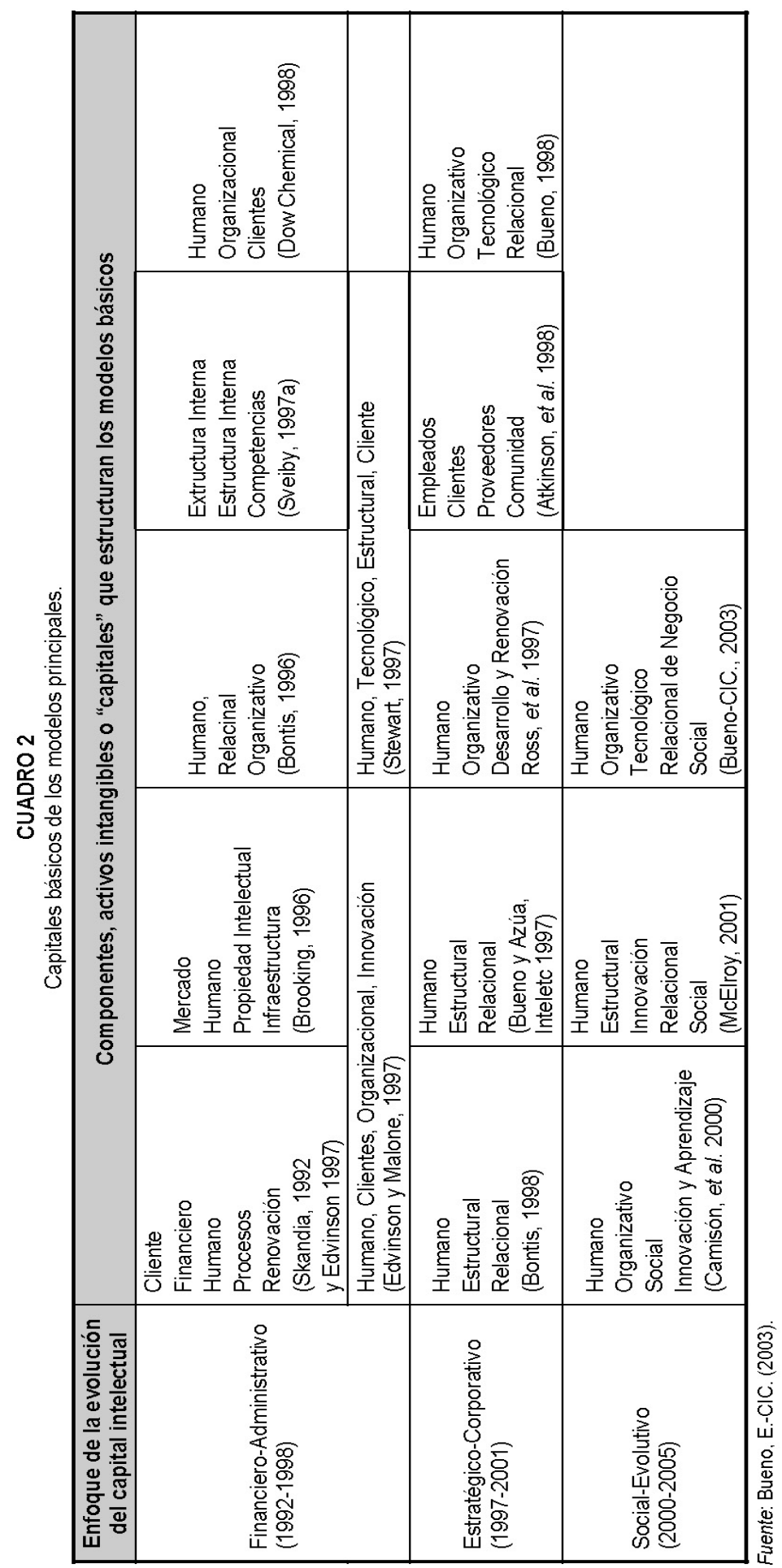


Una vez presentado este conjunto de modelos de medición y gestión del Capital Intelectual, es conveniente avanzar en este campo de investigación y considerar las posibles semejanzas y diferencias entre ellos. Este ejercicio comparativo facilita la identificación de las tendencias básicas seguidas en la formulación de los modelos de medición y gestión del Capital Intelectual y, por tanto, permite el desarrollo de planteamientos que proporcionan una visión integradora de sus elementos esenciales, tal y como se recoge en el Cuadro 3.

\section{CUADRO 3}

Comparativa de los principales modelos de capital intelectual.

\begin{tabular}{|c|c|}
\hline Semejanzas & Diferencias \\
\hline $\begin{array}{l}\text { - Emergencia y desarrollo a mediados y finales } \\
\text { de la década de los noventa. } \\
\text { - Interés en los activos organizativos intangibles } \\
\text { basados en conocimiento } \\
\text { - Vinculación íntima de los modelos con la estra- } \\
\text { tegia. } \\
\text { - Finalidad doble: Interna o de gestión; y externa } \\
\text { o de información a terceros. } \\
\text { - Utilización de indicadores como criterio de me- } \\
\text { dición y gestión de los activos organizativos } \\
\text { intangibles. } \\
\text { - Ausencia de auditorías y de indicadores están- } \\
\text { dares indicadores estándares. } \\
\text { - Heterogeneidad en las unidades de medida de } \\
\text { los indicadores y falta de asignación de valor } \\
\text { financiero al Capital Intelectual. }\end{array}$ & $\begin{array}{l}\text { - Nomenclatura: Ausencia de un lenguaje común. } \\
\text { - Perspectiva financiera. El Cuadro de Mando In- } \\
\text { tegral de Kaplan y Norton incluye dicha pers- } \\
\text { pectiva; mientras que otros modelos no la in- } \\
\text { cluyen, como por ejemplo, el modelo Intelect, el } \\
\text { de A Brooking o el de Sveiby, entre otros. } \\
\text { - Enfoque estático/dinámico. Algunos modelos (Mo- } \\
\text { delo de Bontis; Modelo Intelect; Roos, Roos, } \\
\text { Edvinsson y Dragonetti) hacen referencia a las } \\
\text { interrelaciones que existen entre los componen- } \\
\text { tes del Capital Intelectual. } \\
\text { - Enfoque presente/futuro. Existen propuestas que } \\
\text { reconocen explícitamente la perspectiva de pre- } \\
\text { sente y la de futuro, caso del modelo de Skan- } \\
\text { dia y de Intelect. } \\
\text { - Propuesta de agrupación de los indicadores por } \\
\text { niveles. En concreto, el Intellectual Asset Moni- } \\
\text { tor (Sveiby, 1997) propone tres tipos de indica- } \\
\text { dores para cada uno de los componentes de Cl } \\
\text { que analiza: indicadores de crecimiento y reno- } \\
\text { vación, indicadores de eficiencia e indicadores } \\
\text { de estabilidad. }\end{array}$ \\
\hline
\end{tabular}

Fuente: Bueno, E.-CIC (2003) y elaboración propia.

En líneas generales, todos estos esfuerzos de modelización han incluido, cada uno desde su propia perspectiva, aquellos componentes e indicadores más significativos que tienen una clara incidencia en el proceso de toma de decisiones estratégicas de la empresa.

La evolución de esta dinámica de medición, revelación o información y gestión del Capital Intelectual en España se inició con la propuesta del Modelo Intellect (Euroforum, 1998) que se incluye como uno de los primeros referentes a nivel nacional. La razón fundamental es que dicho Modelo ha servido de ejercicio de reflexión e integración, teniendo un impacto destacado en las comunidades académica y empresarial por desarrollar una propuesta que recoge unos criterios y estructura 
generalmente aceptados a través del análisis de los modelos existentes más relevantes (Bueno, Jericó y Salmador, 2000). Dicho proceso reflexivo e integrador ha evolucionado hacia el Modelo Intellectus (Bueno-CIC, 2003), que viene teniendo un evidente impacto a nivel nacional e internacional, con aplicaciones y referencias muy diversas a nivel económico y organizativo, según se presentará en los epígrafes 5 y 6 .

Previamente, en el epígrafe 4 que recogemos a continuación, se van a mostrar algunas de las definiciones más conocidas y aceptadas de Capital Intelectual, deducidas del breve análisis efectuado sobre los modelos principales. Éstas van a actuar de prolegómeno y de base de la propuesta de un concepto integrador de Capital Intelectual, como síntesis de la génesis y evolución comentada, en coherencia con la lógica semántica y cognitiva con la que se ha venido desarrollando este trabajo.

\section{PROPUESTA DE CONCEPTO DEL CAPITAL INTELECTUAL}

Las definiciones existentes que integran el concepto de Capital Intelectual muestran cierto grado de heterogeneidad, tal y como queda se recoge en el Cuadro 4 . Se corrobora así el estado aún emergente del concepto y la necesidad de seguir investigando a la vez que cooperando o trabajando en red, entre académicos y profesionales, con el fin de crear una comunidad científica o un programa de investigación de aceptación conceptual generalizada entre aquéllos. 


\section{CUADRO 4}

Definiciones básicas del Capital Intelectual.

\begin{tabular}{|l|l|}
\hline \multicolumn{1}{|c|}{ AUTORES } & \multicolumn{1}{c|}{ DEFINICIÓN } \\
\hline List (1841) & $\begin{array}{l}\text { El resultado de la acumulación de los descubrimientos, invenciones, me- } \\
\text { joras, perfeccionamientos y esfuerzos de todas las generaciones que } \\
\text { nos han precedido" (Capital Intelectual de la raza humana) }\end{array}$ \\
\hline Kendrick (1961) & $\begin{array}{l}\text { El resultado de las inversiones en el descubrimiento y difusión del co- } \\
\text { nocimiento productivo. }\end{array}$ \\
\hline Bontis (1996) & $\begin{array}{l}\text { La relación de causalidad entre el capital humano, relacional y orga- } \\
\text { nizativo. }\end{array}$ \\
\hline Brooking (1996) & $\begin{array}{l}\text { La combinación de activos intangibles que permiten a la empresa fun- } \\
\text { cionar. }\end{array}$ \\
\hline Bradley (1997) & $\begin{array}{l}\text { La habilidad de transformar el conocimiento y los activos intangibles en } \\
\text { recursos creadores de riqueza para las empresas y para los países. }\end{array}$ \\
\hline Edvinsson y Malone (1997) & $\begin{array}{l}\text { La posesión de conocimientos, experiencia aplicada, tecnología organi- } \\
\text { zativa, relaciones con los clientes y destrezas profesionales que pro- } \\
\text { porcionan una ventaja competitiva en el mercado. }\end{array}$ \\
\hline Stewart (1997) & $\begin{array}{l}\text { El conocimiento, la información, la propiedad intelectual y la experien- } \\
\text { cia que pueden ser utilizados para crear nueva riqueza. }\end{array}$ \\
\hline Sveiby (1997) & $\begin{array}{l}\text { La combinación de activos intangibles que generan crecimiento, reno- } \\
\text { vación, eficiencia y estabilidad en la organización. }\end{array}$ \\
\hline Lev (2001) & $\begin{array}{l}\text { Representa las relaciones principales, generadoras de activos intangi- } \\
\text { bles, entre innovación, prácticas organizativas y recursos humanos. }\end{array}$ \\
\hline Bueno (2002) & $\begin{array}{l}\text { Representa la perspectiva estratégica de la "cuenta y razón" de los in- } \\
\text { tangibles de la organización. }\end{array}$ \\
\hline Mølbjerg-Jørgensen (2006) & $\begin{array}{l}\text { Desde una perspectiva filosófica, entendido como conocimiento sobre } \\
\text { el conocimiento, creación de conocimiento y apalancamiento del mismo } \\
\text { en valor social o económico. }\end{array}$ \\
\hline $\begin{array}{l}\text { Recursos estratégicos organizativos que permiten a la misma crear va- } \\
\text { lor sostenible, pero que no están disponibles a un gran número de em- } \\
\text { presa (escasez). Generan beneficios potenciales futuros que no pue- } \\
\text { den ser tomados por otros (apropiabilidad) y que no son imitables por } \\
\text { los competidores o sustituibles por otros recursos. No son transferibles } \\
\text { debido a su carácter organizativo. }\end{array}$ \\
\hline
\end{tabular}

No obstante, definiciones propuestas recientemente, como la de Kristandl y Bontis (2007), ponen de manifiesto cómo existen teorías que pueden contribuir a integrar las diferentes aportaciones realizadas, como es la Teoría de los Recursos y Capacidades. Asimismo, se pueden deducir algunos elementos que permiten la configuración de un concepto basado en sus aspectos o caracteres principales. Entre otros, cabe destacar los siguientes:

- Expresa un proceso de acumulación, propio del contenido económico de capital. Indica, en consecuencia, el valor de la riqueza acumulada derivada del conocimiento o de un conjunto de activos de naturaleza intangible.

- Explica una naturaleza intangible o de activos generados por el conocimiento puesto en acción en la organización. 
- Combina activos de naturaleza intangible, los cuales generan nuevo conocimiento. Éste se transforma en competencias empresariales o en la creación de ventaja competitiva.

- Está, en cierta medida, oculto o no suele representarse en los estados contables.

- Genera valor a la empresa y representa la nueva riqueza de las organizaciones y de las naciones.

- Integra diferentes activos intangibles, dinamizados por una estrategia basada en flujos de conocimiento o en actividades intelectuales en la organización.

Con estos argumentos, como síntesis del análisis conceptual efectuado, se propone el siguiente concepto de Capital Intelectual como forma de contribuir o de construir con la lógica semántica y cognitiva ya anunciada en este nuevo campo económico-empresarial:

“Acumulación de conocimiento que crea valor o riqueza cognitiva poseída por una organización, compuesta por un conjunto de activos de naturaleza intangible o recursos y capacidades basados en conocimiento, que cuando se ponen en acción, según determinada estrategia, en combinación con el capital físico o tangible, es capaz de producir bienes y servicios y de generar ventajas competitivas o competencias esenciales para la organización en el mercado".

\section{MODELO INTELLECTUS DE MEDICIÓN Y GESTION DEL CAPITAL INTELECTUAL}

El argumento desarrollado acerca del Capital Intelectual presenta un sentido estratégico a partir de su consideración como herramienta práctica que permite la identificación y medición de los activos intangibles que aportan valor a la organización.

Con este propósito se configura el Modelo Intellectus, fruto de la participación y consenso de diferentes agentes públicos y privados dentro del seno del Foro del Conocimiento e Innovación "Intellectus" como plataforma de transferencia y reflexión del Instituto Universitario de Investigación IADE de la Universidad Autónoma de Madrid.

En este sentido, el Modelo Intellectus pretende facilitar un marco ordenado para la reflexión y puesta en marcha de iniciativas cuyo fin sea la gestión del Capital Intelectual de la organización. Para ello, se cuenta con una estructura de partida que recoge las mejores prácticas existentes en el panorama internacional dentro de esta temática, además de una serie de aportaciones derivadas de los talleres de trabajo llevados a cabo en el citado Foro Intellectus.

Muchas de las contribuciones (Brooking, 1996; Roos et al., 1997; Edvinsson, 1992; entre otras), ostentan un determinado perfil de especialización o un elemento que les hace más apropiado para un colectivo de usuarios, ya sean empresas dedicadas a la I+D, entidades financieras, consultoras, etc. Por su parte, el Modelo Intellectus por sus numerosas y diversas aplicaciones ha mostrado un grado de ajuste 
importante tanto al sector privado como al público (CajaMadrid, Ayuntamiento de Leganés, Cellerix, Instituto de Estudios Fiscales, CajaMurcia, Agencia Tributaria de España, CIRTA,...), según se presenta en el siguiente epígrafe, dimensionando el Capital Intelectual y aportando una base sólida para la reflexión estratégica de la organización.

La estructura del Modelo Intellectus es arborescente y, por tanto, flexible y adaptable en contenidos y detalle, construyéndose bajo la premisa de cinco capitales que interactúan en la dinámica de configuración del potencial organizativo, siguiendo la pauta básica generalmente aceptada descrita por el Capital Humano, el Capital Estructural y el Capital Relacional (ver Figura 2). Pauta propuesta tras considerar diversas investigaciones confirmatorias en relación a las posibles dimensiones y subcomponentes del Capital Intelectual (Bontis, Keow y Richardson, 2000; Martos, Fernández-Jardon, \& Figueroa, 2008; Moon \& Kym, 2006).

FIGURA 2

Estructura del Modelo Intellectus.

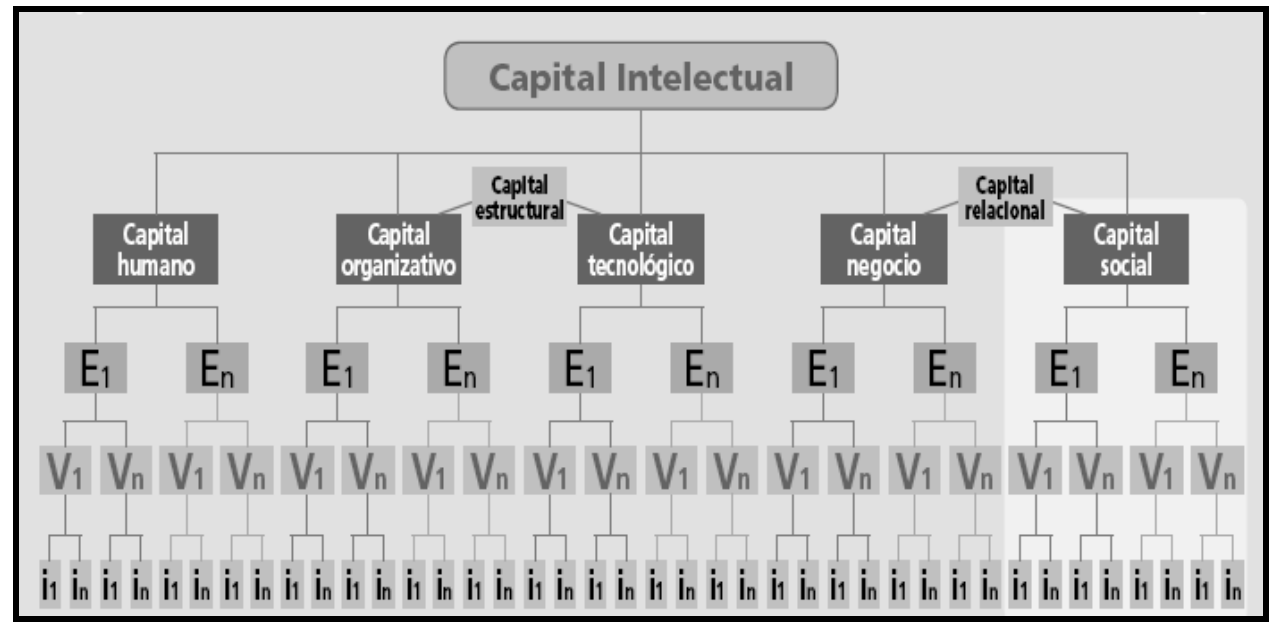

Fuente: Bueno, E.-CIC. (2003).

Para el Modelo Intellectus la composición del Capital Intelectual, tal y como muestra la Figura 3), viene determinada, en primer lugar por el Capital Humano, como aquellas actitudes, aptitudes y capacidades que están en línea con los retos y valores de la organización. En esta línea, ya el Premio Nobel Becker (1964) consideraba el Capital Humano como un factor económico primario en su estudio sobre el conocimiento, refiriéndose a éste como habilidades y destrezas que las personas van adquiriendo a lo largo de su vida, bien sea por medio de estudios formales, como las escuelas, o por conocimientos informales, que son lo que adquieren las personas por medio de la experiencia, y es aquí precisamente donde Becker (1964) pone más énfasis, donde señala que muchos trabajadores elevan su productividad aprendiendo en el puesto de trabajo nuevas técnicas o perfeccionando las antiguas. En segundo lu- 
gar, destaca el Capital Estructural, compuesto a su vez por dos subconjuntos como son el Capital Organizativo y el Capital Tecnológico, división legitimada por sus diferentes implicaciones para la gestión, el primero asociado al ámbito estructural de los diseños, procesos y cultura, y el segundo vinculado con el esfuerzo en $\mathrm{I}+\mathrm{D}$, el uso de la dotación tecnológica y los resultados de la citada I+D. Finalmente, se encuentra el Capital Relacional también dividido en dos, por un lado, el Capital Relacional de Negocio en el que tienen acomodo los flujos de información y conocimiento de carácter externo atados al negocio (proveedores, clientes-usuarios, aliados,...) y, por otro, el Capital Relacional Social, cuyo encuadre se aproxima al marco de relaciones fuera del ámbito del negocio (compromiso social, imagen pública, reputación, prestigio, acción social,...).

FIGURA 3

Elementos del Modelo Intellectus.

\begin{tabular}{|c|c|c|c|c|}
\hline \multicolumn{5}{|c|}{ Componentes } \\
\hline Capital humano & $\begin{array}{c}\text { Capital } \\
\text { organizativo }\end{array}$ & $\begin{array}{c}\text { Capital } \\
\text { tecnológico }\end{array}$ & Capital negocio & Capital social \\
\hline \multicolumn{5}{|c|}{ Elementos } \\
\hline $\begin{array}{l}\text { Valores y } \\
\text { aptitudes. } \\
\text { Aptitudes. } \\
\text { Capacidades. }\end{array}$ & $\begin{array}{l}\text { Cultura } \\
\text { Estructura. } \\
\text { Aprendizaje } \\
\text { Organizativo. } \\
\text { Procesos. }\end{array}$ & $\begin{array}{l}\text { Esfuerzo en I+D+i. } \\
\text { Dotación } \\
\text { tecnológica. } \\
\text { Propiedad intelec- } \\
\text { tual e industrial. }\end{array}$ & $\begin{array}{l}\text { Relaciones con } \\
\text { clientes. } \\
\text { Relaciones con } \\
\text { proveedores. } \\
\text { Relaciones con } \\
\text { aliados. } \\
\text { Relaciones con } \\
\text { competidores. } \\
\text { Relaciones con } \\
\text { medio de } \\
\text { comunicación e } \\
\text { imágene } \\
\text { corporativa. } \\
\text { Relaciones con } \\
\text { las instituciones } \\
\text { de promoción y } \\
\text { mejora de la } \\
\text { calidad. }\end{array}$ & $\begin{array}{l}\text { Relaciones con } \\
\text { accionistas, } \\
\text { instituciones } \\
\text { e inversores. } \\
\text { Relaciones con } \\
\text { Administraciones } \\
\text { Públicas. } \\
\text { Relaciones con la } \\
\text { defensa del medio } \\
\text { ambiente. } \\
\text { Relaciones } \\
\text { sociales. } \\
\text { Reputación } \\
\text { corporativa. } \\
\text { Otras relaciones } \\
\text { con la sociedad. }\end{array}$ \\
\hline \multicolumn{5}{|c|}{ Variables } \\
\hline \multicolumn{5}{|c|}{ Indicadores (Categoría y niveles) } \\
\hline
\end{tabular}

Fuente: Bueno, E.-CIC. (2003).

Todos ellos generan una composición sistémica que permite obtener una panorámica o "stock temporal" de los activos intangibles que posee la organización, generándose así la información necesaria para la toma de decisiones con sentido estratégico para el apropiado gobierno del conocimiento donde se ubican los procesos de actuación que tratan de mejorar la situación existente del Capital Intelectual de la organización. 
Este planteamiento es realmente significativo dado que posiciona al Capital Intelectual en la vertiente táctico-estratégica y al gobierno del conocimiento en la técnico-operativa, diferenciando claramente los "qués" de los "cómos", el stock del flujo, los elementos de los procesos. Sin lugar a dudas, el ejercicio de identificación y medición del Capital Intelectual no resulta un fín en sí mismo sino más bien un requisito imprescindible para actuar coherentemente desde la gestión de los activos que aportan valor a las organizaciones por su relación con las capacidades que mueven el conjunto de recursos tangibles disponibles.

No obstante, el marco del Capital Intelectual y su expresión a través del Modelo Intellectus facilita, entre otros, los siguientes aspectos:

- Identificar, a través de una reflexión estructurada, los activos intangibles claves de la organización.

- Establecer indicadores numéricos de medición que posibiliten un seguimiento periódico de la evolución de tales activos.

- Definir directrices para gestionar estratégicamente el conjunto de intangibles organizativos.

- Complementar la información obtenida en la memoria anual (balance, cuenta de resultados e informe de gestión) con datos sobre los activos intangibles e indicadores no financieros.

\section{PRINCIPALES APLICACIONES Y EXPERIENCIAS DEL MODELO INTELLECTUS}

La utilidad de los modelos de Capital Intelectual se valida y mejora con su uso y aplicación en diferentes esferas, agentes y contextos. Centrándonos en el Modelo Intellectus, éste cuenta con un historial de utilización muy rico y diverso, que contrasta y confirma su marco general y su ajuste específico a los diversos requerimientos.

En las 34 ocasiones de las que se tiene constancia de su aplicación por el IADE, éstas se pueden clasificar en dos grupos generales, a saber:

(a) Sector público. Los principales motivos por los que se desarrollan los proyectos de Capital Intelectual en este caso se asocian a las estrategias de modernización en las Administraciones Públicas, cuyo propósito es avanzar en la mejora de la gestión pública con instrumentos que evidencien los activos intangibles que se encuentran en el seno de los servicios públicos. En este sentido, y ante el nuevo marco de actuación de las Administraciones Públicas en el ámbito de los países de la OCDE, y en especial de la Unión Europea, aparece como indispensable el planteamiento de una nueva función pública basada en la información y el conocimiento.

Este enfoque supone una redefinición de funciones, agentes y procesos de gestión basados en el conocimiento y su dirección; incluyendo la necesidad de identi- 
ficar y poner en acción los activos intangibles que poseen como fuente de creación de valor. Explicitar los intangibles y medir su potencial debería conducir hacia un cambio en la orientación y sentido del servicio público.

Ante tal escenario, las Administraciones Públicas, como estructuras basadas en conocimiento, están considerando varias metodologías a través de modelos conceptuales que cada vez más introducen elementos de contraste que permiten identificar, gestionar y medir el impacto de los intangibles en las organizaciones.

Dentro de las aplicaciones del Modelo Intellectus en este ámbito público se podrían citar los casos de la Agencia Tributaria de España (AEAT), el Instituto de Estudios Fiscales (IEF), el Ayuntamiento de Leganés y Madri+d ${ }^{1}$, éste último concretando una interesante aportación del Capital Intelectual a la estrategia regional de la Comunidad de Madrid (proyecto europeo SPRING 2) en materia de emprendedores.

En una primera instancia, las aplicaciones de Capital Intelectual para los organismos del Ministerio de Hacienda, AEAT e IEF, tuvieron un sentido exploratorio orientado hacia el ajuste del Modelo Intellectus a la realidad del sector público. Se recogieron todas las especificidades tanto terminológicas como estructurales que permitían la comprensión y utilidad del modelo para un gestor público.

Por otra parte, la experiencia con el Ayuntamiento de Leganés revela la importancia del Capital Intelectual como campo de gran potencial para el avance y desarrollo en la línea no sólo de la gestión más completa de los activos que poseen las Administraciones Locales, sino también de una labor de comunicación más integral y con mayor recorrido en el mensaje al ciudadano.

Entre otros factores a destacar, en el caso de Leganés, cabe hacer mención expresa a los ejes que vertebran gran parte del esfuerzo en la gestión de los intangibles, a saber, el ámbito de la calidad y la revolución provocada por el proyecto alrededor de la administración electrónica, centrada en el denominado "Leganés: Ciudad Digital".

Este panorama abre un frente de actuación que puede liderarse desde el enfoque de las tecnologías de la información y las comunicaciones además de las certificaciones y auto-evaluaciones de calidad como herramientas que argumenten el discurso sobre el gobierno del conocimiento, evidenciando requerimientos generales en el tratamiento de la información externa y la generación de un entorno virtual o plataforma coherente con el registro, accesibilidad, uso y difusión de conocimiento en la organización.

Por tanto, el informe de Capital Intelectual basado en el Modelo Intellectus sirve no como documento sino como dinamizador de actitudes para la valorización del componente intangible del Ayuntamiento a modo de eje prioritario de actuación dentro del Plan Estratégico municipal.

En el caso del Capital Intelectual y el uso del Modelo Intellectus en el contexto regional, cabe resaltar su ajuste a los requerimientos de análisis coyuntural multidisciplinar que necesita el fenómeno emprendedor, tanto desde el lado de las actitudes como de la formación, la cultura, las redes y los recursos regionales existentes. 
En este sentido, en el plano territorial, más allá de los planteamientos industriales y técnicos, el motor del desarrollo socioeconómico ha estado vinculado al ser humano y sus relaciones, como sistema de creación y transferencia de información y conocimientos básicos en el proceso acumulativo que lleva a la innovación, bienestar y calidad de vida.

Desde este punto de vista, la valorización de los elementos se plantea desde alejamiento del plano tangible y físico para focalizarse en la identificación, análisis, manejo y gestión de los recursos intangibles, caracterizados por su contribución a la capacidad innovadora y competitiva de los agentes que se relacionan en los denominados sistemas de I+D+i. Ocasionalmente, las posibilidades de la nueva economía y la Sociedad de la Información aportan un importante grado de conectividad y conexión entre los diferentes territorios y agentes, que en un proceso de globalización muy acentuado, aparecen como nodos de un sistema mundial de conocimiento.

De esta forma, la peculiaridad de este enfoque conlleva un exponencial giro en el intercambio de flujos de información y conocimiento, que en su carácter combinatorio basado en el networking, experimentan un efecto acelerador en el aprendizaje general de la sociedad, en la identificación y desarrollo de oportunidades para el emprendimiento, conociendo y por ende, teniendo condiciones para aprender nuevas realidades, modelos, etc.

(b) Sector privado. Esta realidad presenta un elenco de motivaciones más diversas, aludiendo a la necesidad de un mayor nivel competitivo a través del aprovechamiento del talento y el conjunto de capacidades organizativas que tienen como objetivo desarrollar labores de innovación y mejora.

Considerando este ámbito, las aplicaciones han resultado multisectoriales, incluyendo: Cajamadrid, Caixa Popular y Cajamurcia, en el caso de la banca; Cellerix, Imbiosis, Biomol Informatics, Decantum Systems, Noricum, Volatil Absorver, Protein Alternatives, Amplicel y Project Science to Technology, en el terreno de la biotecnología y salud; Ergodomus e Intecdom, en domótica; Gamma Solutions, Betatick, Vaelsys, Quota Solutions, Almira Labs, Itera, Versátil e ICA2, en el sector de la Tecnologías de la Información y las Comunicaciones; Eurogalley Aircrafts Interiors, Zonnect, Indizen Optical Technologies, Greenback y CI2T, en Ingeniería; y finalmente, Advantia Plus, Cimera y Cirta en medioambiente.

De una forma más específica, en el caso de la banca la motivación hacia este tipo de iniciativas viene generalmente determinada por su filosofía de negocio ligada a la valoración de los activos que se disponen, considerando también el marco del Capital Intelectual a modo de ejercicio complementario al momento de configuración de su documentación corporativa anual.

Por otro lado, si se trata de las empresas de base tecnológica (EBTs), el Modelo Intellectus se convierte en una elemento de análisis estratégico básico para las empresas donde el conocimiento tecnocientífico es la base de su negocio. Así, la proyección del I+D se argumenta bajo parámetros apropiados para evaluar el potencial del Know How que posee la compañía. 
Dentro del citado contexto del colectivo de estas EBTs, en una primera instancia y para el caso del Capital Humano, el marco de análisis se concentra en el diagnóstico de las credenciales que presenta, principalmente, el equipo promotor. Credenciales vinculadas en un primer lugar, con el mundo académico (artículos, comunicaciones, etc.), que le otorgan prestigio dentro de la comunidad científica. Por otra parte, credenciales de transferencia asociadas a la realización de proyectos y contratos con diferentes instituciones, lo que refleja el impacto, en las organizaciones del sector público y privado, de sus aportaciones. En este Capital Humano también se busca la identificación de experiencias empresariales o emprendedoras previas, además de la existencia de perfiles especializados para la gestión.

Respecto al Capital Estructural, el diagnóstico de las EBTs responde fundamentalmente a la existencia de una estructura organizativa definida, la formalización de procesos y el esfuerzo en I+D+i. Todos ellos, aspectos que permiten dilucidar el grado de madurez de la organización, y, por ende, la superación del voluntarismo y posible caos de las primeras fases. Es más, se logra diferenciar si la orientación hacia el "I+D como negocio" es real o se quiere hacer "un negocio rápido" con un resultado concreto de la I+D acumulada durante etapas pre-empresa.

Finalmente, en cuanto al Capital Relacional, se analizan las características del mercado objetivo y el soporte derivado del marco de contactos y alianzas. Todo ello se complementa con el ámbito social en el que se valoran activos tan significativos hoy en día como la notoriedad, imagen y reputación.

Así, el Capital Intelectual permite disponer de una metodología apropiada para generar una instancia clave para la reflexión que lleva consigo pasar de la "creación de una empresa" al "desarrollo de un negocio".

En definitiva, de las diversas aplicaciones se desprenden una serie de retos para la investigación que ya están siendo afrontados desde diferentes grupos e instituciones. Los derroteros se sitúan en la senda de la determinación de ciertos índices de capital intelectual basados en sistemas de puntuación para las diferentes variables según capitales, fases empresariales, sectores y coyuntura, cuya aplicación permita generar posibles rankings de organizaciones, regiones, etc., e incluso facilitar enormemente los ejercicios de valoración de empresas en procesos donde se requiere conocer de forma integral el valor del conjunto de activos disponibles.

\section{CONCLUSIONES Y FUTURAS LÍNEAS DE INVESTIGACIÓN}

De las ideas expuestas y de la observación de la génesis, evolución y aplicaciones del Capital Intelectual se puede concluir que, a pesar de haber transcurrido algo más de quince años desde la aparición del concepto, aún es pronto para aceptar que el mismo sea generalmente aceptado por la comunidad académica y profesional. Este trabajo pretende ser una pequeña contribución que intenta animar a la reflexión, al estudio y al debate científico en este terreno. El objetivo es continuar construyendo este posible paradigma emergente y seguir desarrollando el concepto de referencia desde perspectivas actuales de naturaleza epistemológica y de socio- 
logía del conocimiento. Todo ello persigue la formalización de un marco conceptual y de un programa de investigación que incorpore la heurística necesaria para su desarrollo científico.

Es claro que existen grandes perspectivas de desarrollo, las cuales se materializan en la aparición de nuevas revistas científicas especializadas en Capital Intelectual de ámbito internacional, así como en la observación del aumento significativo de los trabajos de investigación, las tesis doctorales y las publicaciones, tanto científicas como de divulgación, en las que el Capital Intelectual es el sujeto y objeto de investigación. Las citadas contribuciones hacen referencia tanto a las organizaciones en general como a tipologías específicas o vinculadas a territorios socioeconómicos, como son las ciudades, regiones, clusters y naciones. La evolución continúa y el desarrollo del concepto y de modelos explicativos de Capital Intelectual, integrando y desarrollando nuevos componentes, será cuestión a destacar en los próximos años, esperando la construcción exitosa de este sugerente y necesario nuevo término económico, característico de la actual Sociedad y Economía del Conocimiento.

En consecuencia, para lograr lo expuesto anteriormente y en coherencia con la observación de las debilidades metodológicas y las dificultades explicativas y operativas del nuevo concepto y de sus modelos de análisis, parece conveniente abordar nuevas líneas de investigación. Entre ellas, se podrían considerar alguna de las propuestas siguientes:

- Concretar la estructura del modelo lógico de capital intelectual, con una aceptación general y refutada no sólo de los componentes sino también de los elementos y variables principales que lo integran, expresivos del valor que se desea revelar.

- Elaborar un índice sintético o un "mapa" de variables e indicadores principales y que respondan a una "medida cuantitativa" relevante y representativa del conjunto de elementos y activos intangibles. Esta medida puede facilitar futuros estudios comparativos y evolutivos.

- Disponer de series históricas significativas sobre las aplicaciones llevadas a cabo, con el fin de poder hacer análisis transversales y de naturaleza estratégica, sobre el impacto de los procesos de gestión del Capital Intelectual en la creación de valor de la organización, una vez definidos los objetivos que puedan medir dicha creación u otros que sean de interés medir por la dirección de la empresa.

- Considerar la necesidad de dinamizar el modelo de análisis del Capital Intelectual, con la incorporación de un nuevo componente o capital, como puede ser el Capital de Emprendimiento e Innovación, estudiando las relaciones causaefecto y la generación de procesos evolutivos y de mejora en los otros componentes o capitales básicos del modelo. 


\section{REFERENCIAS BIBLIOGRÁFICAS}

ABOODY, D. y B. LEV (1998): "The Value Relevance of Intangibles: The Case of Software Capitalization", Journal of Accounting Research, $n^{\circ} 36$, pp. 161-191.

ABRAMOVITZ, M.y DAVID, P. (1996): "Technological Change and the Rise of Intangible Investments: The US Economy's Growth-path in the Twentieth Century”, Employment and Growth in the Knowledge-based Economy, OCDE, Paris.

American Society for Training and Development (2000): Developing and Measuring Intellectual Capital, US.

AMIR, E. y B. LEV (1996): "Value-Relevant of Nonfinancial Information: The Wireless Communication Industry", Journal of Accounting and Economics, $\mathrm{n}^{\circ} 22$, pp. 2-30.

ANDRIESSEN, D. (2004): "IC valuation and measurement: Classifying the state of the art", Journal of Intellectual Capital, vol. 5(2), pp. 230-242.

ATKINSON, A. A.; WATERHOUSE, J. H. y WELLS, R. B. (1997): "A stakeholder approach to strategic performance measurement", Sloan Management Review, pp. 25-37.

BALL, R y BROWN, P. (1968): "An empirical evaluation of accounting income numbers", Journal of Accounting Research, vol. 6, pp. 159-178.

BECKER, G. S. (1964): Human Capital: A Theoretical and Empirical Analysis, with Special Reference to Education, Chicago, University of Chicago Press.

BELL, D. (1973): The coming of post-industrial society: A venture in social forecasting, Basic Books, New York.

BENTHAM, J. (1789): An Introduction to the Principles of Morals and Legislation, London.

BONTIS, N. (1996): "There's a price on your head: Managing intellectual capital strategically", Business Quarterly, Summer, pp. 41-47.

BONTIS, N. (1998): "Intellectual Capital: An Exploratory Study that Develops Measures and Models", Management Decision, vol. 36, $\mathrm{n}^{\circ}$ 2, pp. 63-76.

BONTIS, N.; KEOW, W. y RICHARDSON, S. (2000): "Intellectual capital and the nature of business in Malaysia", Journal of Intellectual Capital, vol. 1, n.1, pp. 85-100.

BRADLEY, K. (1997): "Intellectual capital and the new wealth of nations", Business Strategy Review, vol. 8, n'.4, pp. 33-44.

BROOKING, A. (1996): Intellectual Capital, International Thomson Business Press. Thomson Learning Europe, London.

BUENO, E. (2002): "Dirección estratégica basada en conocimiento: Teoría y práctica de la nueva perspectiva", en Morcillo, P. y Fernández Aguado; J. (2002): Nuevas Claves para la Dirección Estratégica, Ed. Ariel, Barcelona.

BUENO, E.-CIC. (2003): Modelo Intellectus: Medición y Gestión del Capital Intelectual, Documento Intellectus, $n^{\circ} 5$, CIC-IADE (UAM), Madrid.

BUENO, E. (2005): "Génesis, evolución y concepto del Capital Intelectual: Enfoques y modelos principales", Capital Intelectual, $\mathrm{n}^{\circ} 1$.

BUENO, E.; JERICÓ, P. y SALMADOR, M. P. (2000): "Experiencias en medición del capital intelectual en España: El Modelo Intelect” en Bueno, E. y Salmador, M. P. (eds.): Perspectivas sobre Dirección del Conocimiento y Capital Intelectual, I. U., Euroforum Escorial, Madrid.

BARNEY, J. (1991): "Firm resources and sustained competitive advantage", Journal of Management, vol. 17, $\mathrm{n}^{\circ} 1$, pp. 99-120.

CAMISÓN, C.; PALACIOS, D. y DEVECE, C. (2000): "Un nuevo modelo para la medición de Capital Intelectual en la empresa: El modelo NOVA", $X$ Congreso Nacional ACEDE, Oviedo, pp. 3-5, Septiembre.

CETIN, F. (2000): "Managing and measuring Intellectual Capital. Theory and Practice", Seminar in Business Strategy and International Business, University of Technology, Helsinki.

Cluster del Conocimiento (2000): El ABC del Capital Intelectual para PYMES, País Vasco. 
COMTE, A. (1848): Discours sur l’ensemble du positivisme, París.

DAVENPORT, T. H. y PRUSAK, L. (1998): Working Knowledge: How Organizations Manage What They Know. Cambridge, MA: Harvard Business School Press.

DOW CHEMICAL (1998): Dow Chemical's Annual Report, US.

DRUCKER, P. (1965): The Future of Industrial Man, New American Library, London.

EDVINSSON, L. (1997): "Developing intellectual capital at Skandia", Long Range Planning, vol. 30 (3), pp. 366-373.

EDVINSSON, L. y MALONE, M. S. (1999): El capital intelectual, Gestión 2000, Barcelona.

EUROFORUM (1998): El Modelo Intelect, Instituto Universitario Euroforum Escorial, Madrid.

KENDRICK, J.V. (1961): "Some Aspects of Capital Measurement", The American Economic Review, vol. 51, $\mathrm{n}^{\circ} 1$, pp. 102-111.

KRISTANDL, G. y BONTIS, N. (2007): "Constructing a definition for intangibles using the resource based view of the firm", Management Decision, vol. 45(9), pp. 1510-1524.

LEV, B. (1989): "On the Usefulness of Earnings and Earnings Research: Lessons and Directions form two Decades of Empirical Research“, Journal of Accounting Research, vol. 27, pp. 153-192.

LEV, B. (2001): Intangibles: Management, Measurement and Reporting, The Brookings Institution Press.

LEV, B. y SOUGIANNIS, T. (1996): "The Capitalization, Amortization and Value-Relevance of R\&D", Journal of Accounting and Economics, vol. 21, pp. 107-138.

LEV, B. y ZARAOWIN P. (1999): "The Boundaries of Financial Reporting and How to Extend Them", Journal of Accounting Research, vol. 37, pp. 353-385.

LIST, F. (1841): Das Nationale System der Politischen Okonomie, Tubinga.

MACHLUP, F. (1980): Knowledge: Its Creation, Distribution and Economic Significance, vol. 1, Princeton University Press, Princeton, New Jersey.

MARSHALL, A. (1890): Principles of Economics, Mac Millan and Co. London.

MCELROY, M.W. (2001): Social innovation capital draft, Macroinnovation Associates, July, pp. 1-14.

MARTOS, M. S.; FERNÁNDEZ-JARDON, C. M. y FIGUEROA, P. F. (2008): "Evaluación y relaciones entre las dimensiones del capital intelectual: El caso de la cadena de la madera de Oberá (Argentina)", Intangible Capital, vol. 4(2), pp. 67-101.

MEGNA, P. y M. KLOCK (1993): "The impact of Intangible Capital on Tobins Q in the Semiconductor Industry", American Economic Review, vol. 83, pp. 265-269.

MERITUM PROJECT (2002): Guidelines for managing and reporting on intangibles, Fundación Airtel-Vodafone, Madrid.

MILL, J. S. (1848): Principles of Political Economy, Cambridge.

MØLBJERG-JØRGENSEN, K. (2006): "Conceptualising Intellectual Capital as a Language Game and Power", Journal of Intellectual Capital, vol. 7, n 1, pp. 78-92.

MOON, Y. J. y KYM, H. G. (2006): "A model for the value of intellectual capital", Canadian Journal of Administrative Sciences, vol. 23(3), pp. 253-269.

MOURITSEN, J.; LARSEN, H.T. y BUKH, P.N. (2001): "Understanding intellectual capital statements: Designing and communicating knowledge management strategies", en Bontis, N.y Chong, C. (Eds.): World Congress on Intellectual Capital Readings. ButterworthHeinemann, London.

PETTY, R. y GUTHRIE, J. (2000): "Intellectual capital literature review, measuring, reporting and management", Journal of Intellectual Capital, vol. 1(2).

REED, K. K.; LUBATKIN, M. \& SRINIVASAN, N. (2006): "Proposing and testing an intellectual capital-based view of the firm", Journal of Management Studies, vol. 43(4), pp. 867-893.

ROOS, J.; ROOS, G.; DRAGONETTI, N. y EDVINSSON, L. (1998): Intellectual Capital: Navigating in the New Business Landscape, New York University Press, New York.

SAINT ONGE, H. (1996): "Tacit Knowledge: The key to the strategic alignment of Intellectual capital", Strategy \& Leadership, vol. 24 (2), pp. 10-14.

SENIOR, N.W. (1836): Outline of the Science of Political Economy, London. 
SIDGWICK, H. (1883): The Principles of Political Economy, Cambridge.

SKANDIA (1992): Skandia's Annual Report, Sweden.

STEWART, T.A. (1997): Intellectual Capital. The New Wealth of Organizations, Nicolas Brealey Publ., London.

SVEIBY, K. (1997): The New Organizational Wealth, Berret-Koehler, New York.

WERNERFELT, B. (1984): "A resource-based view of the firm", Strategic Management Journal, vol. 5, $\mathrm{n}^{\circ} 2$, pp. 171-180.

TOBIN, J. (1969): "A general equilibrium approach to monetary theory", Journal of Money Credit and Banking, vol. 1(1), pp. 15-29.

VIEDMA, J.M. (2001): "IICBS Innovation Intellectual Capital Benchmarking System", en Bontis, N. (Ed.): World Congress on Intellectual Capital Readings, Butterworth Heinemann, pp. 243-265. 
